\title{
A study to compare maternal and perinatal outcome in early vs. late onset preeclampsia
}

\author{
Pooja Wadhwani, MD¹, Pradip Kumar Saha, MD, MAMS, FIMSA', Jaswinder Kaur Kalra, MD¹, \\ Shalini Gainder, MD', Venkataseshan Sundaram, MD, DM² \\ Departments of ${ }^{1}$ Obstetrics and Gynecology, ${ }^{2}$ Neonatology, Postgraduate Institute of Medical Education and Research (PGIMER), Chandigarh, India
}

\section{Objective}

The risk factors, clinical trends, and maternal and fetal health of early- and late-onset preeclampsia have not been adequately studied. We examined the effects of early- and late-onset preeclampsia on maternal and perinatal outcomes as well as the known risk factors of preeclampsia.

\section{Methods}

One hundred and fifty women with preeclampsia were consecutively enrolled in each group. Those who developed preeclampsia before 34 weeks of gestation were identified as having early-onset preeclampsia, while those who developed at 34 weeks or later were identified as having late-onset preeclampsia. Maternal and perinatal outcomes were compared between groups.

\section{Results}

Compared with the late-onset group, the early-onset group had higher rates of abruptio placentae (16\% vs. $7.3 \%$; $P=0.019)$, but there was no intergroup difference in the composite maternal outcomes. A significantly higher number of women with early-onset preeclampsia developed severe features during the disease course, and most required treatment with antihypertensive drugs. Late-onset preeclampsia was more prevalent among primigravid mothers. Babies born to mothers with early-onset preeclampsia had a significantly higher rate of adverse outcomes.

\section{Conclusion}

These study findings indicate that women with early-onset preeclampsia had more adverse outcome than those with late-onset preeclampsia, but the difference was not statistically significant. There were more babies with adverse perinatal outcomes in the early-than late-onset group.

Keywords: Early-onset preeclampsia; Late onset preeclampsia; Maternal outcome; Perinatal outcome

\section{Introduction}

Preeclampsia is defined by new-onset hypertension and either proteinuria or end-organ dysfunction after 20 weeks of gestation in previously normotensive women [1]. It involves multiple organ systems and is unique to pregnancy. Preeclampsia complicates $2-10 \%$ of pregnancies [2] and represents one of the most common causes of maternal mortality and severe maternal morbidity. The offspring of mothers with preeclampsia are also at increased risk of neonatal morbidity and mortality [3-6]. Hence, the early detection and management of the disease are necessary to decrease its global burden.

According to new terminology (ACOG 2014), preeclampsia without evidence of end-organ damage is termed pre-
Received: 2019.08.19. Revised: 2019.10.21. Accepted: 2019.11.07. Corresponding author: Pradip Kumar Saha, MD, MAMS, FIMSA Department of Obstetrics and Gynecology, Postgraduate Institute of Medical Education and Research (PGIMER), Sector 12,

Chandigarh 160012, India

E-mail: pradiplekha@yahoo.co.in

https://orcid.org/0000-0002-3200-4124

Articles published in Obstet Gynecol Sci are open-access, distributed under the terms of the Creative Commons Attribution Non-Commercial License (http://creativecommons. org/licenses/by-nc/3.0/) which permits unrestricted non-commercial use, distribution, and reproduction in any medium, provided the original work is properly cited.

Copyright $\odot 2020$ Korean Society of Obstetrics and Gynecology 


\section{Obstetrics \& Gynecology Science}

Pooja Wadhwani, et al. Outcomes of early- versus late-onset preeclampsia

eclampsia without severe features [1]. However, the presence of end-organ damage defines severe preeclampsia. In recent years, a new disease classification based on onset timing consisting of early-onset preeclampsia (EO-PE) occurring before 34 weeks of gestation and late-onset preeclampsia (LO$\mathrm{PE})$ occurring at or after 34 weeks of gestation has gained attention [7-9]. The diagnostic criteria are the same for EO-PE and LO-PE; in fact, this simple division has better prognostic implications than mild vs. severe terminology [10].

Few retrospective studies have defined the outcomes of EO-PE and LO-PE and compared them with the outcomes of pregnancies without preeclampsia, whereas studies comparing the risk factors, clinical trends, laboratory parameters, and composite outcomes of maternal and fetal health in the 2 groups are limited. The clinical parameters and associated prognostic implications of the two groups may reflect the different mechanisms of the disease.

This prospective study examined the effects of EO-PE and LO-PE on composite maternal and perinatal outcomes and identified the similarities and differences in laboratory parameters and clinical presentations of the 2 groups.

\section{Materials and methods}

This study was performed in the Department of Obstetrics and Gynecology of the Post Graduate Institute of Medical Education and Research, Chandigarh, India, from July 2016 to October 2017. The total of 300 women with preeclampsia who attended the outpatient department and labor room were recruited. Of these 300 preeclamptic women, 150 women were prospectively recruited in each subgroup and written consent was taken from all participants.

The study was approved by our institute's Ethics Committee (INT/IEC/2017/774) and Research Committee. The study subjects diagnosed with preeclampsia who were supervised and delivered at the Department of Obstetrics and Gynecology were included in this study. Preeclampsia was defined according to American College of Obstetrics and Gynecology criteria [1]. We recruited patients with preeclampsia with or without severe features. Only subjects with confirmed gestation and disease onset timing (preeclampsia) were included. One hundred and fifty eligible patients were consecutively recruited in each group (Fig. 1). Those who developed preeclampsia before 34 weeks of gestation were identified as having EO-
PE, while those who developed preeclampsia at or beyond 34 weeks of gestation were identified as having LO-PE. Patients with chronic kidney disease, heart disease, severe anemia (hemoglobin $<7 \mathrm{~g} / \mathrm{dL}$ ), anti-phospholipid antibody syndrome, or a history of drug abuse were excluded from the study.

Maternal and fetal monitoring was performed according to standard guidelines. Labetalol was given as an antihypertensive. All patients with preeclampsia and severe features were admitted to the hospital. Maternal clinical, hematological, and biochemical monitoring was performed at regular intervals. Fetal surveillance in the form of a biophysical profile, non-stress test, and Doppler ultrasonography was performed. If severe features developed, the pregnancy was terminated at 34 weeks and magnesium sulfate was given during delivery. Continuous intrapartum fetal monitoring was performed.

\section{Comparison of maternal and perinatal outcomes}

The following maternal and perinatal outcomes were compared between groups:

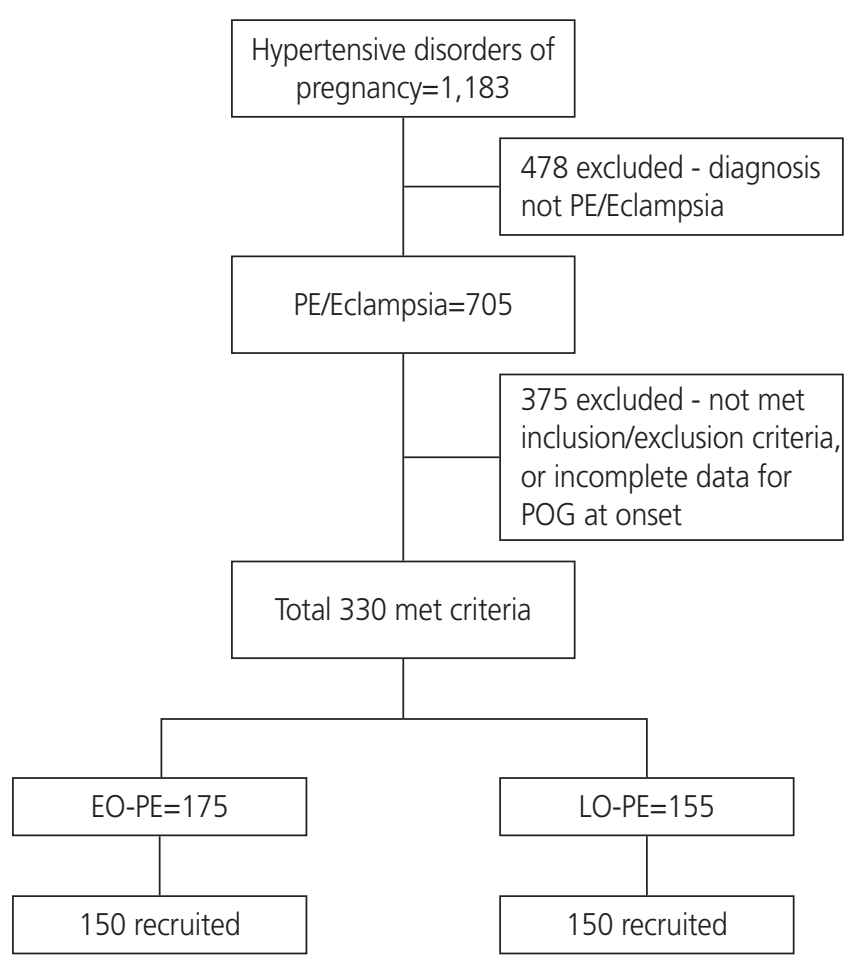

Fig. 1. Consort flow chart showing sample size selection. PE, preeclampsia; $P O G$, period of gestation; EO-PE, early-onset preeclampsia; LO-PE, late-onset preeclampsia. 


\title{
Obstetrics \& Gynecology Science
}

\author{
Vol. 63 , No. 3, 2020
}

i. Adverse maternal outcomes such as hemolysis, elevated liver enzymes, low platelet count (HELLP) [1] syndrome, eclampsia [1], abruptio placentae, postpartum hemorrhage, acute kidney injury [11], venous thromboembolism, cerebrovascular accident, pulmonary edema, and disseminated intravascular coagulopathy were compared between groups;

ii. Adverse perinatal outcomes such as small for gestational age (SGA), stillbirth (intrauterine fetal death after 24 completed weeks of pregnancy) low birth weight, 5-minute Apgar score $<7$, acidosis at birth $(\mathrm{pH}<7.1)$, neonatal intensive care unit (ICU) admission, and the development of complications such as intraventricular hemorrhage, hypoxicischemic injury, respiratory distress syndrome, and necrotizing enterocolitis were compared between the groups. Abnormal umbilical artery Doppler findings (increased systolic/diastolic ratio for that period of gestation, absent end-diastolic flow, and reverse end-diastolic flow) were also compared between groups;

iii. Differences in laboratory parameters in preeclamptic women in the 2 groups;

iv. Difference in the development of imminent and severe features in the 2 groups;

v. Rate of labor induction;

vi. Cesarean section rate. Neonates were classified as SGA when they had a birth weight of less than the 10th percentile for gestational age. Lubchenko charts were used to convert this classification into small for gestational dates [12]; and

vii. Total number of neonates with complications (composite neonatal outcome).

\section{Statistical analysis}

Discrete categorical data are presented as number or percentage (\%), continuous data assumed to be normally distributed are shown as mean and standard deviation, and skewed data are shown as median and interquartile range. The normality of the quantitative data was checked by the Kolmogorov-Smirnov test of normality. Student's $t$-test or the Mann-Whitney $U$ test was used to compare the 2 groups depending upon the normality of the data. Proportions were compared using the $\chi^{2}$ or Fisher's exact test depending on their applicability for the 2 groups. The statistical tests were 2 -sided and performed at a significance level of $a=0.05$. The analyses were conducted using SPSS Statistics (version 22.0; IBM Corp., Armonk, NY, USA).

\section{Results}

A total of 300 patients (150 patients in each group) were followed until discharge after delivery. Table 1 summarizes the baseline demographic details of women with EO-PE and those with LO-PE.

Table 1. Maternal demographic characteristics in women with early- and late-onset preeclampsia (PE) group

\begin{tabular}{|c|c|c|c|}
\hline Characteristics & EO-PE & LO-PE & $P$-value \\
\hline Age (years) & $27.21 \pm 4.4$ & $26.43 \pm 4.04$ & 0.111 \\
\hline $\mathrm{BMI}\left(\mathrm{kg} / \mathrm{m}^{2}\right)$ & $22.52 \pm 1.95$ & $23.26 \pm 1.46$ & $<0.001$ \\
\hline Primigravida & $64(42.7)$ & $83(55.3)$ & 0.028 \\
\hline Diabetes in pregnancy & $10(6.7)$ & $7(4.7)$ & 0.454 \\
\hline Chronic hypertension & $36(24.0)$ & $24(16.0)$ & 0.083 \\
\hline History of PE in previous pregnancies & $30(20.0)$ & $14(9.3)$ & 0.009 \\
\hline Family history of hypertension & $16(10.7)$ & $13(8.7)$ & 0.558 \\
\hline Systolic blood pressure on admission ( $\mathrm{mmHg}$ ) & & & 0.900 \\
\hline Mean $\pm S D$ & $148.91 \pm 16.07$ & $148.67 \pm 16.93$ & \\
\hline Minimum-maximum & $118-200$ & $110-200$ & \\
\hline Diastolic blood pressure on admission $(\mathrm{mmHg})$ & & & 0.445 \\
\hline Mean $\pm S D$ & $96.87 \pm 10.89$ & $95.88 \pm 11.44$ & \\
\hline Minimum-maximum & $60-120$ & $68-140$ & \\
\hline
\end{tabular}

Values are expressed as mean \pm SD or number (\%). P-value $<0.05$ - statistically significant.

EO, early-onset; LO, late-onset; BMI, body mass index; SD, standard deviation. 


\section{Obstetrics \& Gynecology Science}

Pooja Wadhwani, et al. Outcomes of early- versus late-onset preeclampsia

In this study, there were 144 singleton and 6 twin deliveries in the EO-PE group and 143 singleton and 7 twin deliveries in the LO-PE group. A total of 277 live births and 36 intrauterine fetal deaths were recorded.

Mean gestational age at diagnosis was 30.0 weeks and 36.5 weeks in EO-PE and LO-PE groups, respectively. In the EO-PE group, the mean gestational age at admission was 32.1 weeks, mean gestational age at delivery was 32.6 weeks, and mean duration of hypertension from onset to delivery was 17.83 days. In the LO-PE group, the mean gestational age at admission was 37.1 weeks, mean gestational age at delivery was 37.3 weeks, and mean duration of hypertension from onset to delivery was 5.47 days.

The hematological and biochemical parameters are summarized in Table 2. The mean serum creatinine value in EO-PE and LO-PE groups were $0.64 \pm 0.23 \mathrm{mg} / \mathrm{dL}$ and $0.54 \pm 0.40 \mathrm{mg} / \mathrm{dL}$, respectively; the difference was statistically significant.

A significantly higher number of women with EO-PE than those with LO-PE developed severe features during their disease course and required treatment with antihypertensive drugs $(P=0.01)$. The rate of induction was significantly higher in women with EO-PE than in those with LO-PE $(P=0.045)$. However, the cesarean section rate was similar between groups ( $P=0.354$ ) (Table 3).

A total of 78 women (52\%) with EO-PE developed adverse outcomes versus 66 women (44\%) with LO-PE. The difference was not statistically significant. Comparison of the maternal and perinatal outcomes of the twin gestations re- vealed no significant differences.

Maternal complications are summarized in Table 4. Abruptio placentae was significantly more common in women with EO-PE than in those with LO-PE. Women with EO-PE had a significantly higher number of growth-restricted fetuses than those with LO-PE (54.6\% vs. $40.0 \%$, respectively) and a higher number of fetuses with umbilical artery Doppler abnormalities (32.6\% vs. 9.3\%). The rate of stillbirths was significantly higher in the EO-PE group than the LO-PE group (21.0\% vs. $1.9 \%)$. Also, babies born to mothers with EO-PE had significantly higher adverse outcomes (Table 5).

\section{Discussion}

Although the causes of preeclampsia are unclear, multiple authors have suggested that the pathophysiology of EO-

Table 3. Disease severity correlation, labor and delivery details in early- and late-onset preeclampsia (PE) group

\begin{tabular}{lccc}
\hline Treatment & EO-PE & LO-PE & P-value \\
\hline Antihypertensive therapy & $129(86.0)$ & $111(74.5)$ & 0.012 \\
PE with severe features & $112(74.7)$ & $91(60.7)$ & 0.01 \\
MgSO $_{4}$ therapy & $101(67.3)$ & $68(45.3)$ & $<0.001$ \\
Induction of labor & $104(69.3)$ & $96(64.0)$ & 0.045 \\
Cesarean section & $83(55.3)$ & $75(50.0)$ & 0.354 \\
\hline
\end{tabular}

Values are expressed as number (\%). P-value $<0.05$ - statistically significant.

Table 2. Hematological and biochemical parameters in women with early- and late-onset preeclampsia (PE) group

\begin{tabular}{lccc}
\hline Variable & EO-PE & LO-PE & P-value \\
\hline Hemoglobin $(\mathrm{gm} / \mathrm{dL})$ & $10.92 \pm 1.06$ & $10.91 \pm 1.09$ & 0.962 \\
Platelet count $/ / \mathrm{LL})$ & $181,880 \pm 76,895$ & $192,613 \pm 67,770$ & 0.201 \\
PTI & $96.07 \pm 5.21$ & $97.06 \pm 5.06$ & 0.095 \\
Urea $(\mathrm{mg} / \mathrm{dL})$ & $29.94 \pm 12.71$ & $30.60 \pm 11.68$ & 0.640 \\
Creatinine $(\mathrm{mg} / \mathrm{dL})$ & $0.64 \pm 0.23$ & $0.54 \pm 0.40$ & $<0.001$ \\
Bilirubin $(\mathrm{mg} / \mathrm{dL})$ & $0.80 \pm 1.83$ & $0.63 \pm 0.19$ & 0.263 \\
SGOT (U/L) & $43.40 \pm 46.05$ & $39.52 \pm 29.03$ & 0.490 \\
SGPT (U/L) & $42.75 \pm 43.00$ & $41.88 \pm 36.25$ & 0.364 \\
ALP (U/L) & $261.46 \pm 126.16$ & $250.96 \pm 96.86$ & 0.745 \\
\hline
\end{tabular}

Values are expressed as mean \pm standard deviation. $P$-value $<0.05$ - statistically significant.

EO, early-onset; LO, late-onset; PTI, prothrombin time index; SGOT, serum glutamic oxaloacetic transaminase; SGPT, serum glutamate-pyruvate transaminase; ALP, alkaline phosphatase. 


\section{Obstetrics \& Gynecology Science}

Vol. 63, No. 3, 2020

PE differs from that of LO-PE. Early-onset disease appears to be mediated by the placenta. LO-PE, on the other hand, is mediated by maternal factors and a maternal overreaction to pregnancy [13-15]. Our data show no intergroup difference in mean maternal age, and there were significantly more multiparous women in EO-PE group. This observation is similar to that of a study by Stubert et al. [16]. The frequency of risk factors predisposing an expectant mother to developing diabetes and chronic hypertension did not differ between groups; however, patients with LO-PE had a higher mean body mass index than those with EO-PE as reported by the Valensise et al.'s study [13] of patients with two different hemodynamic states.

A history of preeclampsia in a previous pregnancy is a

Table 4. Maternal complications in women with early- and late-onset preeclampsia (PE) group

\begin{tabular}{lcccc}
\hline Complications & EO-PE $(\mathbf{n}=\mathbf{1 5 0})$ & LO-PE $(\mathbf{n}=\mathbf{1 5 0})$ & Total $(\mathbf{n}=\mathbf{3 0 0})$ & $\boldsymbol{P}$-value \\
\hline Complete HELLP & $8(5.3)$ & $4(2.6)$ & $12(4.0)$ & \\
Partial HELLP & & & & \\
$\quad$ Deranged LFT & 9 & 13 & $22(7.3)$ & \\
Thrombocytopenia & 12 & 19 & $31(10.3)$ & \\
Total HELLP & $29(19.3)$ & $36(24.0)$ & $65(21.6)$ & 0.327 \\
Eclampsia & $26(17.3)$ & $20(13.3)$ & $46(15.3)$ & 0.337 \\
Abruption & $24(16.0)$ & $11(7.3)$ & $35(11.6)$ & 0.019 \\
Pulmonary edema & $4(2.7)$ & $2(1.3)$ & $6(2.0)$ & 0.684 \\
AKI & $7(4.6)$ & $4(2.7)$ & $11(3.7)$ & 0.356 \\
PPH & $2(1.3)$ & $6(4.0)$ & $8(2.7)$ & 0.282 \\
CVA & $0(0.0)$ & $1(0.7)$ & $1(0.3)$ & 1.000 \\
Retinal detachment & $2(1.3)$ & $1(0.7)$ & $3(1.0)$ & 0.624 \\
\hline
\end{tabular}

Values are expressed as number (\%). $P$-value $<0.05$ - statistically significant.

EO, early-onset; LO, late-onset; HELLP, hemolysis, elevated liver enzymes, low platelet count; LFT, liver function test; AKI, acute kidney injury; $\mathrm{PPH}$, post-partum haemorrhage; CVA, cerebrovascular accident.

Table 5. Perinatal outcome parameters in women with early- and late-onset preeclampsia (PE) group

\begin{tabular}{lccc}
\hline Parameters & EO-PE $(\mathbf{n}=\mathbf{1 5 6})$ & LO-PE $(\mathbf{n}=\mathbf{1 5 7})$ & $\boldsymbol{P}$-value \\
\hline Small for gestation age neonates & $83(53.2)$ & $60(38.2)$ & 0.008 \\
Umbilical artery Doppler abnormality & $49(31.4)$ & $14(8.9)$ & $<0.001$ \\
Still births & $33(21.2)$ & $3(1.9)$ & $<0.001$ \\
Live births & $123(78.8)$ & $154(98.1)$ & $<0.001$ \\
Birth weight (kg) & $1.502 \pm 0.58$ & $2.416 \pm 0.59$ & $<0.001$ \\
$<2.5$ & $148(94.9)$ & $87(55.4)$ & \\
$\geq 2.5$ & $8(5.1)$ & $70(44.6)$ & \\
5-min Apgar score $<7$ & $10(6.4)$ & $5(3.2)$ & $<0.074$ \\
Respiratory support & $44(28.2)$ & $5(3.2)$ & $<0.001$ \\
Neonatal acidosis (PH $<7.1)$ & $5(3.2)$ & $2(1.3)$ & 0.144 \\
NICU stay & $34(21.8)$ & $4(2.5)$ & $<0.001$ \\
Days of NICU stay (days) & $10.98 \pm 11.7$ & $6.95 \pm 4.36$ & 0.019 \\
Composite Neonatal outcome & $63(40.4)$ & $20(12.7)$ & $<0.001$ \\
Neonatal death & $8(5.1)$ & $4(2.5)$ & 0.112 \\
\hline
\end{tabular}

Values are expressed as mean \pm SD or number (\%). P-value $<0.05$ - statistically significant.

EO, early-onset; LO, late-onset. 


\section{Obstetrics \& Gynecology Science}

Pooja Wadhwani, et al. Outcomes of early- versus late-onset preeclampsia

known risk factor for recurrent preeclampsia. Our study showed that women with preeclampsia in a previous pregnancy are more likely to develop EO-PE in subsequent pregnancies. The result of the present study differs slightly from those of Li et al. [17], who reported that the incidence of developing early preeclampsia with severe hypertension among women with previous history of preeclampsia was similar to that of women who developed late preeclampsia with severe hypertension.

The hematological and biochemical parameters were comparable between groups and showed no significant difference. Only the difference in mean serum creatinine value in the EO-PE and LO-PE groups $(0.64 \pm 0.23 \mathrm{mg} / \mathrm{dL}$ and $0.54 \pm 0.40 \mathrm{mg} / \mathrm{dL}$, respectively) was statistically significant. The laboratory findings of our study were consistent with those of Li et al. [17]. EO-PE is associated with glomerular endotheliosis, which results in a lower glomerular filtration rate (GFR) and effective renal plasma flow. Serum creatinine is a reliable estimate of GFR. This explains the probable cause of the increased serum creatinine value in women with EO-PE.

Significantly more women with EO-PE than those with LO$P E$ developed severe features and required treatment with antihypertensive drugs and magnesium sulfate. As expectant management was given in women with EO-PE, they were started on antihypertensive therapy to control the blood pressure, whereas women with LO-PE who developed severe hypertension requiring antihypertensive therapy underwent immediate pregnancy termination.

In the present study, $52 \%$ of women with EO-PE versus $44 \%$ of women with LO-PE developed adverse outcomes during follow-up until discharge. Although more mothers with EO-PE than those with LO-PE had adverse outcomes, the difference did not reach statistical significance. There were no maternal deaths in either group, but three women with EO-PE and one with LO-PE required ICU admission. Indications for ICU admission included complications such as pulmonary edema, eclampsia with atypical posterior reversible encephalopathy syndrome. The most common maternal complications were HELLP syndrome, abruptio placentae, and eclampsia in both groups. No significant intergroup difference was noted in adverse maternal outcomes. Consistent with the present study, the rate of antepartum hemorrhage was significantly higher in the study conducted by Lisonkova et al. [18]. Another study published by Pettit et al. [19] concluded that preeclampsia causes significant maternal organ dysfunction (acute kidney injury, abnormal liver function, thrombocytopenia, and neuronal complications) regardless of gestational age at onset. Madazli et al. [20] compared the clinical and laboratory findings and maternal-perinatal outcomes between EO-PE and LO-PE groups and concluded the same. The present study and the above mentioned studies $[19,20]$ highlight the fact that no form of preeclampsia should be considered benign.

The stillbirth rate ( $21.0 \%$ vs. $1.9 \%)$ was significantly higher in the EO-PE group than in the LO-PE group. The results of the present study are consistent with those of other studies $[17,20,21]$. One contributing reason to stillbirth in women with EO-PE may be insufficient placentation and incomplete trophoblast invasion of spiral arteries, which results in decreased blood flow to the fetus and fetal distress. The significantly higher number of patients in the EO-PE group than in the LO-PE group had abnormal umbilical artery Doppler findings (Table 5) may be the reason for the higher stillbirth rate seen in the EO-PE group. Another reason for the high stillbirth rate in the EO-PE group was that the patient refused to undergo a cesarean section due to extreme prematurity.

The neonatal death rate was higher in the EO-PE group than in the LO-PE group, although the difference did not reach statistical significance. This difference from previous studies $[20,21]$ was probably due to the small sample size and short follow-up period (until discharge) of the present study.

Adverse neonatal complications were significantly more common in babies born to mothers with EO-PE. Adverse neonatal outcomes affected 63 patients $(47.7 \%)$ in the EO-PE group versus $20(12.9 \%)$ in the LO-PE group, a difference that was statistically significant. This result is consistent with those of most previous relevant studies $[17,20,21]$.

Gestational age at birth was the most important factor contributing to neonatal outcomes. The greater number of adverse perinatal outcomes in the EO-PE group might have been due to prematurity itself than the severity of preeclampsia. Adverse perinatal outcomes were increased in EO-PE, which is naturally expected considering the mean birth weight of 1.5 and $2.4 \mathrm{~kg}$ in the EO-PE and LO-PE groups, respectively.

The primary strength of our study is that it was prospective and performed in a tertiary care teaching institute with a relatively good number of study subjects. Its main limitation was its short follow-up period. 


\section{Obstetrics \& Gynecology Science}

Vol. 63 , No. 3, 2020

In conclusion, a significantly higher number of women with EO-PE than those with LO-PE developed severe features during their disease course, and most required treatment with antihypertensive drugs. LO-PE was more prevalent among primigravid patients. Perinatal morbidity rates were higher in mothers with EO-PE than in those with LO-PE.

Our findings indicate that women with EO-PE had more adverse outcomes than those with LO-PE, but the difference was not statistically significant. Further studies with larger sample sizes are warranted.

\section{Conflict of interest}

No potential conflict of interest relevant to this article was reported.

\section{Ethical approval}

Approved by Institutional Ethics Committee, PGIMER, Chandigarh (INT/IEC/2017/774).

\section{Patient consent}

The patients provided written informed consent for the publication and the use of their images.

\section{References}

1. American College of Obstetricians and Gynecologists; Task Force on Hypertension in Pregnancy. American College of Obstetricians and Gynecologists and others. Hypertension in pregnancy. Report of the American College of Obstetricians and Gynecologists' task force on hypertension in pregnancy. Obstet Gynecol 2013;122:1122-31.

2. Duckitt K, Harrington D. Risk factors for pre-eclampsia at antenatal booking: systematic review of controlled studies. BMJ 2005;330:565.

3. Basso O, Rasmussen S, Weinberg CR, Wilcox AJ, Irgens $L M$, Skjaerven R. Trends in fetal and infant survival following preeclampsia. JAMA 2006;296:1357-62.

4. Badawi N, Kurinczuk JJ, Keogh JM, Alessandri LM,
O'Sullivan F, Burton PR, et al. Antepartum risk factors for newborn encephalopathy: the Western Australian casecontrol study. BMJ 1998;317:1549-53.

5. Friedman SA, Schiff E, Kao L, Sibai BM. Neonatal outcome after preterm delivery for preeclampsia. Am J Obstet Gynecol 1995;172:1785-92.

6. Vestergaard M, Basso O, Henriksen TB, Østergaard J, Secher NJ, Olsen J. Pre-eclampsia and febrile convulsions. Arch Dis Child 2003;88:726-7.

7. Ness RB, Roberts JM. Heterogeneous causes constituting the single syndrome of preeclampsia: a hypothesis and its implications. Am J Obstet Gynecol 1996;175:1365-70.

8. von Dadelszen P, Magee LA, Roberts JM. Subclassification of preeclampsia. Hypertens Pregnancy 2003;22:143-8.

9. Roberts CL, Ford JB, Algert CS, Antonsen S, Chalmers J, Cnattingius $S$, et al. Population-based trends in pregnancy hypertension and pre-eclampsia: an international comparative study. BMJ Open 2011;1:e000101.

10. Turner JA. Diagnosis and management of pre-eclampsia: an update. Int J Womens Health 2010;2:327-37.

11. Summary of recommendation statements. Kidney Int Suppl (2011) 2012;2:8-12.

12. Lubchenco LO, Hansman C, Boyd E. Intrauterine growth in length and head circumference as estimated from live births at gestational ages from 26 to 42 weeks. Pediatrics 1966;37:403-8.

13. Valensise H, Vasapollo B, Gadgliardi G, Noveli GP. Early and late preeclampsia: two different hemodynamic state in the latent phase of the disease. Hypertension 2008; $52: 873-80$.

14. LaMarca BD, Gilbert J, Granger JP. Recent progress toward the understanding of the pathophysiology of hypertension during preeclampsia. Hypertension 2008; 51:982-8.

15. Brosens IA, Robertson WB, Dixon HG. The role of the spiral arteries in the pathogenesis of preeclampsia. Obstet Gynecol Annu 1972;1:177-91.

16. Stubert J, Ullmann S, Dieterich M, Diedrich D, Reimer T. Clinical differences between early- and late-onset severe preeclampsia and analysis of predictors for perinatal outcome. J Perinat Med 2014;42:617-27.

17. Li XL, Guo PL, Xue Y, Gou WL, Tong M, Chen Q. An analysis of the differences between early and late preeclampsia with severe hypertension. Pregnancy Hypertens 2016;6:47-52. 


\section{Obstetrics \& Gynecology Science}

Pooja Wadhwani, et al. Outcomes of early- versus late-onset preeclampsia

18. Lisonkova S, Sabr Y, Mayer C, Young C, Skoll A, Joseph KS. Maternal morbidity associated with early-onset and late-onset preeclampsia. Obstet Gynecol 2014;124:77181.

19. Pettit F, Mangos G, Davis G, Henry A, Brown MA. Preeclampsia causes adverse maternal outcomes across the gestational spectrum. Pregnancy Hypertens 2015;5:198204.
20. Madazli R, Yuksel MA, Imamoglu M, Tuten A, Oncul M, Aydin $B$, et al. Comparison of clinical and perinatal outcomes in early- and late-onset preeclampsia. Arch Gynecol Obstet 2014;290:53-7.

21. Lisonkova S, Joseph KS. Incidence of preeclampsia: risk factors and outcomes associated with early- versus lateonset disease. Am J Obstet Gynecol 2013;209:544.e112. 